\title{
Comprensión Lectora Superficial, Profunda y Reflexivo-Crítica de un Estudiante de Escuela Nueva
}

${ }^{1}$ Institución Educativa Telepalmeritas
ilmayrivera@gmail.com.co

Como citar: Rivera, I. (2021). Comprensión Lectora Superficial, Profunda y Reflexivo-Crítica de un Estudiante de Escuela Nueva Educación Y Ciencia, (25), e12549. https://doi.org/10.19053/0120-7105. eyc.2021.25.e12549

\section{Resumen}

Este artículo presenta resultados parciales de una investigación cuyo objetivo fue describir y analizar cómo los estudiantes de Escuela Nueva en una institución educativa colombiana, comprenden superficial, profunda, crítica y reflexivamente textos expositivos, con ayudas frías y cálidas del docente y acompañamiento de los padres de familia. Se usó el paradigma interpretativo con enfoque cualitativo, recurriendo a la etnografía educativa. La muestra se conformó con siete nińos de primaria. La información fue recolectada mediante encuestas y aplicación de guías multidisciplinarias en las que, a partir de textos expositivos, se interrogó para movilizar los procesos comprensivos. Para el presente escrito, los resultados caracterizan los procesos de comprensión de un texto expositivo de la guía aplicada a un estudiante del grado quinto. Para la comprensión superficial, el estudiante recuperó información literal usando vocablos sueltos, oraciones cortas y enunciados textuales extensos; en la profunda, vio sentidos ocultos y asoció las lecturas con su contexto sociocultural; en la reflexiva-crítica tomó posturas razonadas. Las ayudas cognitivas y afectivas de la docente e involucramiento de los padres de familia, contribuyeron a la consolidación del conocimiento.

Palabras clave: comprensión superficial, profunda y reflexivo-crítica, estudiante, docente, familia, primaria. 


\title{
Superficial, Profound, and Reflective-Critical Reading Comprehension of a Eementary Student
}

\begin{abstract}
This paper presents partial results of an investigation aimed to describe and analyze how students of Escuela Nueva, in a Colombian elementary, understand superficial, profound, critical, and reflective expository texts, with cold and warm help from the teacher and support from parents. The interpretive paradigm was used with a qualitative approach, using educational ethnography. The sample consisted of seven primary school children. I used surveys and the application of multidisciplinary guides, in which, based on expository texts, I questioned the students for mobilizing comprehensive processes. For this article, the results show the understanding of an expository text of a student in the fifth grade. For superficial understanding, the student retrieved literal information using loose words, short sentences, and extensive textual statements; in the profound, he saw hidden senses and associated the readings with their socio-cultural context; in reflective-critical took reasoned positions. The cognitive and affective aids of the teacher and involvement of parents contributed to the consolidation of knowledge
\end{abstract}

Keywords: superficial, profound and reflective-critical understanding, student, teacher, family, elementary.

\section{Introducción}

A pesar de la amplia producción nacional y extranjera enfocada en la lectura, a nivel de primaria y en ocasiones en ámbitos de Escuela Nueva, son escasos los trabajos que vinculan ayudas cognitivas y afectivas del docente e involucran a los padres de familia. Retomar estos aspectos resulta importante porque posibilita al estudiante incursionar con solvencia en los procesos que convergen en la comprensión lectora.

La lectura es el encuentro de la mente de quien escribe con la de quien lee, de la tinta con el papel, de la luz con el ojo, del ojo con el texto, del mensaje con la curiosidad, de lo neurológico con lo psicológico, del lenguaje con la imaginación, del entendimiento con la reflexión, de la idea con el sentimiento, de la libertad con el placer por conocer; lo cual hace trizas las barreras del tiempo y el espacio.

El artículo presenta los resultados parciales de una investigación que se propuso describir y analizar cómo los estudiantes de una institución educativa colombiana, que desarrolla el modelo Escuela Nueva, comprenden de modo superficial, profundo y reflexivo-crítico un texto expositivo cuando reciben ayudas frías y cálidas del docente y acompańamiento de sus padres. En este texto los resultados se centran en el proceso de un estudiante de grado quinto primaria. Se hace evidente la necesidad de aportar elementos de tipo teórico y pedagógico a los estudios enmarcados en la 
lingüística aplicada, específicamente en el campo de la competencia lectora.

Los referentes teóricos que apoyaron la investigación fueron: procesos involucrados en la comprensión, ayudas del docente, acompañamiento de padres de familia y perspectiva pedagógica Escuela Nueva.

\section{Procesos Involucrados en la Comprensión Lectora}

Para Sánchez (2010) hay tres procesos relacionados a la comprensión de un texto escrito: 1) extraer información explícita para alcanzar la competencia superficial; 2) interpretar la información para lograr la comprensión profunda; 3) reflexionar y valorar el texto para llegar a la comprensión reflexivo-crítica.

\section{Extraer Información Explícita para Alcanzar la Comprensión Superfi- cial}

En la comprensión superficial "el lector entiende lo que dice el texto porque es capaz de seleccionar y organizar la información contenida en él” (Sánchez, 2010, p. 63); si domina el código lingüístico respectivo, identificará contenidos explícitos y, desde luego, construirá un resumen del texto. El lector tiene conocimientos lingüísticos que le permiten saber cómo funcionan los elementos de una lengua en cada discurso. Para activar este proceso en el aula, se recurre a diversas estrategias como parafraseo, mapa conceptual, diagrama, ideario con informaciones puntuales o la pregunta del docente para inducir el reporte de información explícita, entre otras. Cuando se trata de textos multimodales se añaden, a la identificación del contenido explícito, elementos no verbales que también los constituyen (imagen, color, luz, sombra, líneas).

\section{Interpretar la Información para Lograr la Comprensión Profunda}

Comprendida la información explícita del texto, el lector podrá interpretar e inferir, esto es, descifrar sentidos ocultos, lo que no se dice pero que se sobreentiende; asociar, establecer comparaciones, relacionar esos sentidos con el propio entorno e identificar y comprender cómo se relacionan semántica y formalmente los elementos locales que configuran el texto (Avendaño \& González, 2020). Y “si no es suficiente con la información contenida en el texto, el lector apelará a sus conocimientos y llevará a cabo algún proceso de integración entre el texto y lo que ya sabe" (Sánchez, 2010, p. 63).

El leer exige conocer el entramado lingüístico del texto, pero también reactivar capacidades cognitivas consubstanciales a la interpretación. Leer no consiste solamente en reconocer la información explícita, también conlleva develar implícitos. Esto se puede lograr a través del docente que moviliza procesos cognitivos en el estudiante como conjeturar lo que se encontrará en el texto o proponer hipótesis de sentido y verificarlas, y descubrir lo no dicho, pero sobreentendido, mediante asociación, inferencia, analogía, comparación y recontextualización a partir de presaberes. Este 
segundo proceso involucrado en la comprensión muestra que el significado del texto no reside solamente en las palabras, frases, oraciones, etc., también está en la mente del lector, quien lo elabora según su acervo cognitivo, experiencias de vida, contextos socioculturales y modos de pensar, sentir y ensoñar (Avendaño \& González, 2020). Cuando la comprensión profunda se alcanza, el texto entra a formar parte de los conocimientos previos y se integra a ellos (Sánchez, 2010).

Para activar este proceso en el aula conviene invitar al estudiante a reconocer los elementos que estructuran el texto; identificar el género discursivo; desentrañar la temática central y temas conexos, el propósito comunicativo del texto, la polifonía de voces que confluyen; y recontextualizar el texto en el entorno del lector, entre otras acciones.

\section{Reflexionar y Valorar el Texto para Llegar a la Comprensión Reflexi- vo-Critica}

Cuando el lector extrae la información explícita y descubre sentidos ocultos está en la capacidad de emitir juicios de valor. "Es capaz de reparar y resolver posibles inconsistencias entre dos textos o entre varios” (Sánchez, 2010, p. 45). Es tarea del docente incentivar procesos de reflexión y crítica argumentada a través del cuestionamiento y la formulación de interrogantes, exhortando al estudiante a que piense sobre lo que leyó y asuma posiciones razonadas.

Avendańo y González (2020) aducen que el texto no surge de la nada, siempre emerge de otros, de los entornos personales y socioculturales de quien lo gesta, de su cosmovisión, de sus propias miradas, ideologías, creencias, emociones y sentimientos, pues la escritura es un proceso situado. Texto, autor y lector no son entes escindidos, detrás de cada uno de ellos se esconden contextos específicos donde los tres actores se amalgaman en el proceso de comprensión. De esta manera, la relación se hace evidente: el lector construye el sentido a partir de contextos socioculturales propios, del autor y de la época en que se creó la obra, de los objetivos de lectura, de sus actitudes y de su posición en la sociedad.

Los tres procesos mencionados, que están involucrados en la comprensión, convergen en todo acto de lectura, pues se dan de modo simultáneo en la mente del lector. En el momento en el que identifica la información explícita, la persona extrae sentidos ocultos que emergen de sus presaberes y de circunstancias socioculturales propias y del autor, lo cual le posibilita pensar sobre lo leído, evaluar el texto y asumir posturas críticas fundamentadas.

\section{Ayudas del Docente en el Proceso de Comprensión Lectora}

La comprensión lectora demanda acompañamiento de las personas involucradas en el aprendizaje de los estudiantes, por eso el docente tiene responsabilidad en este proceso. Para García (2011), el gran reto de los maestros es acercar y fortalecer en 
los discípulos procesos inferenciales y niveles superiores de lectura. Como señala Colomer (1997): “enseñar a entender un texto ha ido convirtiéndose en el objetivo real de las prácticas escolares y ha permitido experimentar y articular nuevas prácticas para conseguirlo" (p. 4). Esa enseńanza requiere ayudas docentes que "no son solo acciones para movilizar procesos, sino que pueden verse como puentes" (Sánchez, 2010, p. 259), son asistencias que garantizan a los estudiantes alcanzar sus objetivos. Lomas y Tusón (2009) plantean que: "la enseńanza de la lectura es una tarea educativa que a todos afecta” (p. 114), su acompańamiento, tanto en escuela como en casa debe ser constante, afectivo y efectivo. Sánchez (2010) clasifica las ayudas que brindan los docentes en frías y cálidas.

\section{Ayudas Frías}

Las ayudas frías son prácticas docentes usadas para superar los obstáculos que presenta el estudiante. Entre ellas se destacan: descomponer la tarea o plantear una nueva pregunta, son fragmentadas, pues la respuesta del lector llega paso a paso; rellenar huecos, es dar parte de la respuesta para que el estudiante la complete; dar apoyo físico o invitar a releer y retomar algún elemento del texto; y dar una mediación instrumental para facilitar la tarea. "Si él [profesor] propone el uso de una estrategia, estará ayudando a comprender, mientras que cuando la describe, estará enseñando a comprender" (Sánchez, 2010, p. 259)

\section{Ayudas Cálidas}

Se caracterizan porque mantienen la motivación y el compromiso del estudiante con las metas y actúan para controlar emociones que amenacen la realización de una tarea (Sánchez, 2010). Regulan las emociones de los aprendices y los liberan de la angustia. Este tipo de ayudas son poco comunes en el quehacer de la enseńanza. Se destaca el elogio, con él se exalta y reconoce el trabajo. Se busca que los estudiantes continúen el proceso de comprensión pese a sus equivocaciones. Por ejemplo: ¡excelente! ¡muy bien! ¡tienes la razón! Es fructífero alabar y estimular en el momento oportuno, pero sin excesos. También es importante la parsimonia en el procesamiento de la información, para mostrar una actitud comprensiva y tolerante cuando el niño se equivoca o se trunca. Por ejemplo: si no sabes, no te preocupes. Trataremos de hallar la respuesta.

\section{Acompañamiento de Padres de Familia en la Comprensión Lectora}

Las familias son responsables de satisfacer las necesidades biológicas, afectivas y de formación valórica de sus hijos. En el espacio escolar su participación debe ser constante y significativa, que no solo acompañe, sino que sea un motor emocional motivacional en la transformación de sus hijos. Esa colaboración atañe también a 
su participación en procesos involucrados en la comprensión lectora, puesto que su ayuda es esencial y motivadora para sus hijos. A propósito, Robledo (2015) manifiesta: "alienten a los niños y niñas en el esfuerzo, compartan lecturas con ellos y apoyen su proceso de manera colaborativa y natural, como se hace cuando los niños están aprendiendo a hablar” (p. 29).

\section{Perspectiva Pedagógica Escuela Nueva}

Esta mirada pedagógica es una alternativa que reconoce las habilidades de los estudiantes y permite que aprendan haciendo; lo que se aprende en todas y cada una de las fases de la experiencia constituye el valor de esa vivencia (Dewey, 1995). La Escuela Nueva es "una propuesta pedagógica que cuenta con componentes curriculares, organizativos, administrativos, de interacción comunitaria y estrategias didácticas" (MEN, 2010, p. 9). Este modelo está dirigido a escuelas rurales con población dispersa, llamadas "multigrado" o escuelas unitarias que cuentan con un solo docente para los cinco grados de primaria. Colbert (1999) seńala que esta propuesta fomenta el aprendizaje activo, fortalece las relaciones escuela-comunidad, involucra a los padres de familia en el aprendizaje de sus hijos, flexibiliza la promoción del estudiante, fortalece valores y actitudes democráticas y promueve un nuevo rol del profesor como facilitador de procesos de aprendizaje.

\section{Metodología}

La investigación se apoyó en el paradigma interpretativo, pues al estar en contacto directo con la realidad del aula se observó su acontecer cotidiano para así obtener información relevante durante el proceso de comprensión del texto expositivo por parte del estudiante de Escuela Nueva. Se adoptó el enfoque cualitativo, toda vez que este permite aplicar procesos investigativos rigurosos para comprender e interpretar las interacciones humanas o los fenómenos sociales para reconstruirlos, transformarlos y, así, construir nuevo conocimiento. En palabras de Bisquerra (2012), "la aproximación a la realidad se lleva a cabo desde adentro, junto con las personas implicadas y comprometidas en dichas realidades (p. 275). Las fases de la investigación fueron: 1) exploratoria diagnóstica, se elaboró una encuesta con once preguntas de selección múltiple para conocer las percepciones sobre las prácticas lectoras y sus preferencias temáticas; 2) diseño de una unidad didáctica conformada por doce guías modelo Escuela Nueva, que incluyen textos expositivos con temáticas multidisciplinarias, cada guía contiene tres momentos: trabajemos juntos, trabajo individual y trabajo en familia; 3) trabajo con guías Escuela Nueva; 4) descripción y análisis de la aplicación de la guía 10. Conservo mis recursos en un estudiante del grado 5.

Por circunstancias derivadas del COVID-19, este trabajo se hizo de manera virtual y remota, lo cual implicó resignificar la práctica pedagógica a través del uso creativo de herramientas tecnológicas como la grabación de la conversación telefónica 
y audios de WhatsApp. La grabación de la clase se transcribió en el diario de campo.

\section{Resultados y Discusión}

Para sistematizar la información de la fase diagnostica se establecieron las siguientes categorías: momentos de lectura en la escuela, preferencias temáticas, relación del contenido del texto con el entorno sociocultural, inferencia del tema central del texto, socialización de opiniones sobre el texto, ayuda del docente en las dificultades de comprensión y lectura con los padres teniendo como referente a un estudiante del grado quinto.

La fase diagnóstica exploratoria indicó que las prácticas lectoras son frecuentes en la escuela multigrado objeto de este estudio. El estudiante se interesa por la lectura de textos expositivos sobre animales, plantas y astronomía, quizá porque su contexto es rural, durante el recorrido diario de la casa a la escuela se mueve en espacios abiertos y está en contacto con la naturaleza. Además, es dueño de saberes previos sobre algunos de los contenidos elegidos, lo que estimula su curiosidad, favorece la comprensión lectora y consolida su conocimiento. Sus reflexiones son compartidas con sus padres y profesora.

Así mismo, sus vivencias en la escuela y en el hogar configuran un bagaje cognitivo que se retoma y consolida a través de la lectura. Además, lee en la casa con sus padres aprovechando que la modalidad Escuela Nueva involucra significativamente a la familia en el proceso educativo.

La información recabada sirvió para diseñar las guías multidisciplinarias. Estas, a su vez, y gracias a las rutas didácticas que vinculan, permitieron caracterizar y analizar los tres procesos implicados en la comprensión de textos expositivos con ayudas frías y cálidas del docente y el acompañamiento de los padres.

Este artículo muestra los resultados de la Guía 10: Conservo mis recursos diseñada para el grado quinto. A continuación, se describe y analiza cómo el estudiante de este grado alcanza la comprensión superficial, profunda y reflexivo-crítica del texto expositivo La lombricultura, presentado en la guía antes citada; la manera como la docente le proporcionó ayudas frías y cálidas; y el acompañamiento de los padres durante el proceso.

Para el posterior análisis de resultados de la aplicación de la guía se grabó la conversación telefónica de una clase. Luego, se realizó una transcripción del diálogo [C1]. De igual manera, se numeró cada línea del diálogo, lo que permitió detallar aspectos relevantes de los procesos involucrados en la comprensión lectura y las ayudas frías y cálidas dadas por la docente. Para el diálogo entre docente y madre de familia se asignó el código M1. 


\section{Extraer Información Explícita para Alcanzar la Comprensión Superfi- cial}

Antes de iniciar la lectura del texto expositivo La lombricultura, el estudiante observó algunas imágenes impresas relacionadas. Luego, el diálogo empezó con la formulación de preguntas para provocar comentarios del estudiante, esto suscitó un clima de interés marcado por la interacción comunicativa. Leyó el texto en voz alta y detectó léxico desconocido, ante lo cual se le invitó a deducir el significado por el contexto de su contenido. Para Avendaño y González (2020), el acto de leer exige conocer el entramado lingüístico del texto, si el lector domina la estructura de la lengua respectiva podrá identificar los contenidos explícitos.

Para asegurar la comprensión superficial, la guía incluyó una serie de preguntas pertinentes para generar diálogo pedagógico. La siguiente es la transcripción de la primera parte de la clase entre la docente y el estudiante:

1. Docente: ¿qué se entiende por lombricultura?

2. Estudiante: donde utilizan lombrices para hacer abono

3. Docente: ¡correctoi ¿Qué hacen con ellas?

4. Estudiante: las dejan en un cajoncito, les echan tierrita y ahí les echan desechos orgánicos

5. Docente: imuy bien ${ }_{i}$

6. Estudiante: y la convierten en abono

7. Docente: ¿qué productos se obtienen de la lombricultura?

8. Estudiante: el abono, la carne

9. Docente: ¿cómo se llama el abono?

10.Estudiante: humus

11.Docente: imuy bien $_{i}[\mathrm{C} 1]$

Se evidencia que en el proceso de comprensión superficial, el estudiante no solo recordó sino que entendió el contenido del texto, pues logró recuperar información explícita mediante el uso de palabras sueltas como "humus", oraciones cortas como "donde utilizan lombrices para hacer abono" y enunciados textuales más extensos como "las dejan en un cajoncito, les echan tierrita y ahí les echan desechos orgánicos". Con ello se demuestra según Sánchez (2010) que el alumno puede entender lo que dice el texto gracias a que ha sido capaz de seleccionar y organizar la información contenida en él ( p. 63).

Para que el estudiante fuera más explícito con respecto a la pregunta “qué entiende por lombricultura?” la docente brindó ayudas frías orientadas a descomponer la tarea 
o plantear una nueva pregunta (línea 3). Según Sánchez (2010), "esta ayuda consiste en simplificar la demanda fragmentándola en pasos o piezas que luego vuelven a recomponerse" (p. 268) hasta obtener la respuesta acertada. Los elogios como ayuda cálida sirvieron como motivación y reconocimiento al esfuerzo (líneas 3, 5 y 11), lo que contribuye a conservar el compromiso e interés del niño. Las preguntas tuvieron como propósito explorar la capacidad del estudiante para identificar la información explícita del texto, ya que una vez se identifique el contenido expuesto en el texto, se descubrirán múltiples sentidos implícitos (Avendaño \& González, 2020)

\section{Interpretar la Información para Lograr la Comprensión Profunda}

Como se enfatizó, leer un texto implica conocer el código lingüístico, pero además poner en movimiento habilidades cognitivas inherentes al desentrañe de diversos contenidos implícitos, porque "leer no consiste solamente en reconocer la literalidad del texto, sino en inferir lo que no se dice, pero que se sobreentiende; asociar, establecer comparaciones, relacionar esos sentidos subyacentes con saberes previos y con el propio entorno sociocultural"(Avendaño \& González, 2020, p. 158). Véase el modo como el estudiante lo hizo:

1. Docente: ¿por qué se considera la lombriz roja californiana como una herramienta de trabajo?

2. Estudiante: porque hace abono, ayuda a los árboles.

3. Docente: ¡conoces en tu sector tierras que han sido devastadas por la erosión? Describe como las ves.

4. Estudiante: no señora

5. Docente: tranquilo no pasa nada. Vamos a descubrir la respuesta

6. Docente: ¿`conoces el significado de la palabra erosión?

7. Estudiante: dańan el suelo

8. Docente: ¿qué pasa con el suelo?

9. Estudiante: lo escarban y hacen huecos.

10.Docente: las personas utilizan los potreros para criar...

11.Estudiante: para criar vacas

12. Docente: ¡correctoj, también cuando llueve mucho ¿qué pasa con la tierra?

13.Estudiante: la tierra se desliza.

14.Docente: ¿qué pasa si en nuestras fincas no tenemos un suelo sano?

15. Estudiante: no podemos sembrar

16.Docente: ¿qué pasa si no se puede sembrar? 
17.Estudiante: no habría cosecha

18.Docente: qué significa suelo sano

19.Estudiante: que se puede cultivar

20.Docente: ¿qué otro título le pondrías al texto leído?

21.Estudiante: las lombrices

22.Docente: podrías ser más específico teniendo en cuenta que el texto se refiere a una lombriz con características especiales. Puedes regresar al texto y observar la infografía.

23.Estudiante: la lombriz roja. [C1]

Las respuestas del niño evidencian que logra hacer inferencias a partir del texto, muestran que generó procesos de asociación derivados de los presaberes adquiridos en su contexto rural. Con esos interrogantes, la docente problematizó el conocimiento y movilizó en el estudiante su capacidad de deducir, evocar y relacionar significados para descubrir sentidos ocultos en el texto. Para Sánchez (2010), cuando la comprensión profunda se alcanza, el texto pasa a formar parte de los conocimientos previos y se integra a ellos. Las acciones pedagógicas orientadas a promover procesos interpretativos también contribuyen a enriquecer el acervo cognitivo del estudiante.

Durante el proceso de lectura, la docente acompañó al estudiante con ayudas frías como: descomponer la tarea, donde la respuesta se consigue paso a paso a través del planteamiento de nuevas preguntas (líneas 6 y 8); rellenar huecos, que consiste en dar parte de la respuesta para que el estudiante la complete (línea 10); dar apoyo físico, invitándolo a retomar la infografía del texto (en la línea 22 la docente sugiere observar la infografía del texto). Cuando el estudiante manifestó desconocer una respuesta, la docente le ofreció una ayuda cálida denominada parsimonia en el procesamiento de la información, es decir, tuvo una actitud comprensiva y tolerante cuando estudiante ignoró la respuesta. En la línea 5 se aprecia la expresión "tranquilo no pasa nada" De igual manera, recurrió al elogio (línea 12). Este tipo de ayuda fue eficaz para regular las emociones del estudiante frente a los obstáculos en la comprensión, para el caso, miedo ante el desconocimiento de la respuesta, angustia e incluso llanto.

\section{Reflexionar y Valorar el Texto para Lograr la Comprensión Reflexi- vo-Crítica}

Cuando el lector extrae la información explícita y descifra sentidos ocultos del texto, puede emitir juicios de valor. Entonces, es imperioso activar este proceso mediante la problematización del conocimiento; hacer cuestionamientos, formular preguntas para que piense sobre lo que lee; evaluar, asumir posturas críticas argumentadas; y entender que ningún texto surge del vacío, pues la escritura es un proceso situado en determinados contextos personales y socioculturales. Además, resulta de la cosmovisión del autor, de sus ideologías, creencias, emociones y sentimientos. Esta 
búsqueda o "buceo" en el tejido textual le permite al lector develar los contextos para pensar sobre ellos y aportar sentido (Avendaño \& González, 2020).

1. Docente: ¡crees que puede haber agricultura sin lombrices? Justifica tu respuesta.

2. Estudiante: no, porque el abono no sale tan bueno"

3. Docente: ¿qué pasa si el abono no sale tan bueno?

4. Estudiante: las plantas no van a crecer

5. Docente: ¿te parece adecuada la forma como las personas de tu sector cuidan el suelo? Explica tu respuesta.

6. Estudiante: no, porque talan mucho

7. Docente: recuerda que al tumbar los árboles no solamente cambia el paisaje a la vista, también se ven afectados muchos animales que allí vivían.

8. Docente: al tumbar las plantas ¿qué ocurre con el suelo?

9. Estudiante: se seca

10.Docente: recuerda la palabra que aprendimos hace un momento la...

11.Estudiante: ¡se erosionan!

12. Docente: ¡qué bien!

13. Docente: ¿consideras la lombricultura como una opción para la alimentación de seres vivos? ¿Por qué?

14.Estudiante: sí, porque a las gallinas les gusta

15. Docente: ¿los seres humanos la pueden consumir?

16.Estudiante: no creo, debe saber raro

17.Docente: te cuento que la lombriz roja californiana sí puede ser consumida por el ser humano, para ello requiere de un proceso de limpieza, de tal forma que pueda ser utilizada por él. De hecho, se utiliza como carne para hamburguesa. [C1]

El fragmento anterior describe cómo el estudiante abordó el proceso de reflexión y crítica; asumió posturas fundadas en vivencias alimentadas por un contexto sociocultural agropecuario; activó saberes previos sobre el manejo de fertilizantes al cuestionar la eficacia de productos agroquímicos empleados para mejorar las cosechas, al señalar que su baja calidad impide el crecimiento de la planta; tomó una posición frente al cuidado del suelo y advirtió que la tala de árboles lo está destruyendo. Sus posturas críticas argumentadas emergen de su experiencia de vida en la ruralidad.

Respecto a las ayudas brindadas por la docente, se observa su apoyo con ayudas frías como descomponer la tarea (líneas 3 y 15) y dar pistas (líneas 10 y 11). Además, lo elogió con calidez para exaltar su respuesta (línea 12). 
A partir de la lectura de un solo texto, expositivo en este caso, es posible reactivar en el estudiante los tres procesos involucrados en la comprensión de lectura, toda vez que como lo advirtieron Avendaño y González (2020), estos confluyen en cualquier acto lector y ocurren de modo simultáneo en la mente. Cuando se reconoce la información superficial, se infieren sentidos ocultos que afloran de las circunstancias socioculturales propias y del texto, gracias a los presaberes y a procesos cognitivos como la asociación, la inferencia, la analogía, la comparación y la recontextualización. Lo anterior permite reflexionar, evaluar y tomar posiciones críticas razonadas. Sin embargo, al retomarlos en el aula de clase, cada proceso se explora de modo lineal por organización y como estrategia didáctica.

\section{Acompañamiento de Padres de Familia en los Procesos de Comprensión}

En el caso estudiado se contó solo con el acompañamiento de la madre porque el padre cumplía compromisos laborales. Durante el desarrollo de la guía se le preguntó al estudiante:

1. Docente: ¿cuál es el miembro de la familia que más te ayuda en tus actividades escolares?

2. Estudiante: con mi mamá, ella me pone atención y me explica lo que no entiendo

3. Docente: ¿¿de qué forma te colaboró tu familia en el desarrollo de la guía?

4. Estudiante: me ayudó respondiendo las preguntas

5. Docente: ¿cómo te sientes cuando ella te acompaña?

6. Estudiante: ¡bien; , me gusta que ella me acompañe. [C1]

El nińo reconoce que la madre es el miembro de la familia que más lo acompaña en el proceso lector, específicamente con las preguntas que no comprende. Es ella quien más le presta atención y le explica lo que no entiende, lo cual imprime seguridad a su desempeño académico. A continuación se describe el diálogo entre la docente y madre de familia [M1]

1. Docente: sobre la lectura correspondiente a la lombricultura. ¿En qué le colaboró a su hijo?

2. Madre de Familia: en realidad no le ayudé mucho pues no conocía el tema

3. Docente: ¡el tema fue nuevo para los dos?

4. Madre de Familia: sí señora, pero pedí ayuda con otras personas de la casa y buscamos información en internet.

5. Docente: ¿̨le aportó información nueva la lectura sobre la lombricultura?

6. Madre de Familia sí señora, hay temas que no se sabían y compartiendo con el niño queda información nueva. 
7. Docente: ¿considera importante su acompañamiento en las actividades escolares de sus hijos?

8. Madre de Familia: sí señora, porque hay temas que los niños no entienden, nosotros los podemos ayudar, a veces ellos se distraen muchos y se está pendiente para que desarrollen sus trabajos. [M1]

La madre de familia comentó que desconocía el tema tratado en la guía y para ayudar a su hijo buscó información con otras personas y en internet. Considera que acompañarlo en el proceso lector le permitió fortalecer saberes y mantener una comunicación abierta y más afectiva con el niño. Al respecto, Robledo (2015) advierte que:

no se trata de que los padres asuman el papel de docentes ni utilicen las mismas metodologías de enseńanza, sino que alienten a los nińos y nińas en el esfuerzo, compartan lecturas con ellos y apoyen su proceso de manera colaborativa y natural (p. 29).

La emergencia sanitaria vivida durante el proceso de esta investigación propició el acompañamiento de los padres de familia en las actividades de comprensión lectora, amplió los tiempos para compartir e impactó favorablemente en la actitud de los hijos frente a sus responsabilidades no solamente académicas, sino del hogar.

\section{Conclusiones}

La fase exploratoria mostró que el estudiante se interesa más por textos expositivos sobre animales, plantas y astronomía, quizá, debido al contexto rural en el que vive. Esta información propició la conformación del corpus y el diseño de la ruta didáctica para dinamizar, describir y analizar el modo como abordó los tres procesos involucrados en la comprensión, con ayudas frías y cálidas de la docente y la vinculación de la madre de familia.

Respecto al primer proceso, extraer información explícita para alcanzar la competencia superficial, el nińo entendió el contenido del texto propuesto y recuperó información literal mediante el empleo de vocablos sueltos, oraciones cortas y enunciados textuales más extensos. Es decir, comprendió la literalidad del texto, la información que aparece en la superficie.

Sobre interpretar la información para lograr la comprensión profunda, las respuestas del estudiante muestran que puede asociar significados gracias a los presaberes adquiridos, pues el entorno nutre su comprensión lectora. Además, las preguntas de la docente coadyuvan en la activación de la capacidad del nińo para evocar, inferir y relacionar hechos, ideas y concepciones propias que permiten descubrir sentidos tácitos. Las acciones pedagógicas y didácticas dinamizaron procesos interpretativos que enriquecieron el bagaje cognitivo del estudiante. 
En relación a reflexionar y valorar el texto para llegar a la comprensión críticoreflexiva, una vez lograda la información explícita y descifrados los sentidos ocultos, se hace posible emitir juicios de valor, pensar sobre el texto, ponerlo en contextos sociales y culturales propios y asumir posturas argumentadas. La lectura del texto planteado permitió que el estudiante adoptara posiciones sustentadas en experiencias vividas en su entorno agropecuario, en el que temas como los cultivos, la cosecha, los abonos, la tala de árboles, la erosión y la conservación del suelo, le resultan familiares.

Gracias a las acciones didácticas y metodológicas elegidas y desarrolladas por la docente, fue posible la caracterización y análisis de los tres procesos vinculados a la comprensión lectora. Así, se destacan como acciones propicias: la selección de textos expositivos de interés para los niños, la organización de breves soportes teóricos, la inclusión de imágenes visualmente llamativas, el cuestionamiento sobre el texto elegido y el planteamiento de incertidumbres para que el estudiante se sumerja en el océano lingüístico, bucee allí, pesque múltiples sentidos, reflexione, burbujee y exprese sus propios puntos de vista. Es decir, esa ruta didáctica también le permitió al estudiante seguir un sendero para incursionar en los tres procesos precitados.

Sobre las ayudas cálidas y frías brindadas por la profesora se destaca su acertado y continuo uso para motivar y asistir el proceso de lectura del estudiante. Estas acciones resultan favorables también en la gestión de emociones, tan necesaria para el aprendizaje, ya que un cerebro angustiado no comprende y mucho menos aprende; no obstante, un docente empático y siempre justo, estará atento a lanzar oportunamente un salvavidas afectivo y efectivo al buzo en dificultades.

La participación de la madre de familia en los procesos de comprensión estrechó los lazos afectivos en el hogar, toda vez que el desarrollo de la comprensión lectora requiere acompañamiento empático y cariñoso. Además, su compromiso se evidenció en la indagación de fuentes vivas y en la web, cuando requirió dar explicaciones sobre temas desconocidos, así ambos (madre e hijo) también adquirieron nuevos conocimientos.

La pandemia causada por el COVID-19 se constituyó en un limitante para el desarrollo de la investigación, debido la ausencia total del contacto directo con los estudiantes y con los padres de familia. No obstante, las circunstancias inherentes a los confinamientos avivaron el pensamiento creativo para continuar el proceso con ahínco y tenacidad. Por último, aún queda abierta la puerta para que otros estudiosos del lenguaje sigan indagando sobre la competencia lectora, ojalá en todos los niveles del sistema educativo.

\section{Referencias}

Avendaño, G., \& González, O. (2020). Una propuesta de plan de clase para dinamizar la lectura crítica. Folios, (52), 155-171.h ttps://doi.org/10.17227/folios.52-10002 
Bisquerra, R. (2012). Metodología de la investigación Educativa. La Muralla

Colbert, V. (1999). Mejorando el acceso y la calidad de la educación para el sector rural pobre. El caso de la Escuela Nueva en Colombia. Revista Iberoamericana de Educación, (20), 107-135. https://rieoei.org/historico/documentos/rie20a04.htm

Colomer, T. (1997). La enseñanza y el aprendizaje de la comprensión lectora. Signos. Teoría y práctica de la educación, (20), 6-15. http://www.xtec.cat/-ilopez15/ materials/comprensiolectora/laensenanzayelaprendizajedelacomprensionlectora. pdf

Dewey J. (1995). Democracia y Educación. Morata

García, P. (2011). Escribir textos expositivos en el aula. Graó

Lomas, C., \& Tusón, A. (2009). Enseñanza del lenguaje, emancipación comunicativa y educativa critica: el aprendizaje de las competencias comunicativas en el aula. Edére

Ministerio de Educación Nacional. (2010). Manual de implementación Escuela Nueva. Generalidades y orientaciones pedagógicas para transición y primer Grado. Tomo I. Ministerio de Educación Nacional.

Robledo, B. (2015). Familias lectoras: Como formar lectores y escritores en el hogar. Tragaluz

Sánchez, M. (2010). La lectura en el aula, Qué se hace, qué se debe hacer, y que se puede hacer. Grao 